\title{
Modeling of Tunneling Current of A Bilayer Armchair Graphene Nanoribbon-Based P-N Junction Diode using Airy Wave-Function
}

\author{
A Fadhillah ${ }^{1}$, I Anjaningsih ${ }^{2}$, S D Ulhaq ${ }^{3}$, M F Fadhillah ${ }^{4}$, Waslaluddin ${ }^{5}$, and E Suhendi ${ }^{6}$ \\ \{Ameliafadhillah57@gmail.com ${ }^{1}$ \} \\ Department of Physics Education, Universitas Pendidikan Indonesia, Bandung, Jawa Barat,
Indonesia ${ }^{1,2,3,4,5}$
}

\begin{abstract}
Armchair Graphene Nanoribbon (AGNR) is one of the Graphene Nanoribbon (GNR)'s types which can be used as a semiconductor that arrange its width. Because of its tiny width, graphene is great to be applied on devices with tunneling electron-based. In this research, the author models the behavior of tunneling current on a bilayer armchair graphene nanoribbon-based p-n junction diode using Airy function. The modeling's result shows that the tunneling current has a relation with bias voltage, temperature, and characteristic of BAGNR. The tunneling current is directly proportional to the forwardbias, the width of the BAGNR, and the intrinsic electric field. It is inversely proportional to the temperature. Its value on BAGNR is bigger than on MAGNR due to the effect of interlayer coupling $\gamma_{0}$ and $\gamma_{1}$ to the energy gap. The tunneling current using the Airy function method and the WKB method have the same tendency and slight different value.
\end{abstract}

Keywords: BAGNR, P-N Junction diode, Tunneling current, Airy function

\section{Introduction}

P-n junction is a basic concept of the current semiconductor devices development such as diodes and transistors. Therefore, proper characterization of $\mathrm{p}-\mathrm{n}$ junction is indispensable to optimize its development in the modern semiconductor devices [1]. The current that generated in the p-n junction can create either from the mechanism of electron diffusion or tunneling. However, this diffusion mechanism requires high enough voltage in its operation. The tunneling mechanism is applied in a Zener diode with the application of a reverse bias. Through this mechanism, Zener diodes can operate at low voltage. To optimize the ability of electronic devices operating at low voltage, a material that has high electron mobility and a tiny band gap is needed. One material that meets these criteria is graphene.

Graphene is a promising material for nanoelectronics field because of its special electrical properties with stable thermodynamic and mechanical properties. Carrier charge mobility in graphene can be increased up to $200,000 \mathrm{~cm}^{2} / \mathrm{Vs}$ [2], while increased the electronic properties of the material on $\mathrm{SiO}_{2}$ substrate only reached $2000-15000 \mathrm{~cm}^{2}$ / Vs [3]. Graphene doesn't have a band gap because the valence band and the conduction band meet at the corner of the first Brillouin zone [4]. This makes the conductivity in graphene difficult to stop and cannot be used in digital electronic applications. To modify the band gap in graphene, various studies have been carried out which then produce Graphene Nanoribbon. The electrical properties and band gap energy in graphene nanoribbon $(\mathrm{GNR})$ can be regulated through their edge and width 
structure. Based on its edge structure, GNR is divided into two types, namely Zigzag Graphene Nanoribbon (ZGNR) and Armchair Graphene Nanoribbon (AGNR). ZGNR is a conductor, while AGNR can be a conductor or semiconductor depending on its width[4],[5]. Current technology is still difficult to produce (monolayer graphene) one layer of graphite. Micromechanical cleavage and graphite exfoliation only produce multilayer graphene. However, graphite, bilayer graphene, and multilayer graphene are derivatives of graphene. Therefore, graphene bilayer is a material that generally has properties such as graphene with easier fabrication and is a useful basis for multilayer graphene [6]. Bilayer graphene is also known as a material whose energy gap can be regulated through an electric field perpendicular to the gate potential [7],[8].

The electrical properties of GNR have been extensively studied and simulated to illustrate their characteristics when applied in electronic devices. As though [9] which modeling the characteristics of tunneling current in $\mathrm{p}$-n junction that made from graphene nanoribbon and carbon nanotubes using the WKB method. This method has been widely known to solve mathematical solutions of various physical phenomena, one of which is the Schrödinger equation. Modeling by [10],[11], [5] about tunneling current in the p-n junction and bipolar transistors [11] that made from GNR. Modeling is done using the Airy function approach method [11],[10]and the transfer matrix method [5],[11], the results are compared with the WKB method. The tunneling current that generated through the Airy function transfer method and the Transfer Matrix method shows the same values, while the WKB method shows lower values. Based on the research [12], the WKB method has a weakness to execute the electron wave equation in the turning point area. Therefore, in this study, the author will model the tunneling current in the $\mathrm{p}-\mathrm{n}$ junction that made of bilayer armchair graphene nanoribbon by using the Airy function approach method and the result will be compared with the WKB method.

\section{Method}

Energy band of the bilayer graphene can be written by mathematical expression in Equation $1[1]$

$$
E(k)=s \frac{\hbar^{2} k^{2}}{2 m^{*}}
$$

$\mathrm{s}=+1$ for conduction band dan $\mathrm{s}=-1$ for valence band, $\hbar$ is the reduced Planck constant, and $m^{*}$ is effective mass. Effective mass can be written mathematically $m^{*}=\frac{\gamma_{1}}{2 v_{f}^{2}} \cdot \gamma_{1}$ is the interlayer coupling whose value $\approx 0.39 \mathrm{eV}[13]$ and $v_{f}$ is the fermi velocity whose value $10^{6}$ $\mathrm{m} / \mathrm{s}$. Transversal momentum of GNR quantized based on its width, therefore $k=\frac{n \pi}{3 w}$ dan $n=$ $\pm 1, \pm 2, \pm 3, \ldots$. With the dimension, width of GNR $w$ than its length $L(w<<L)$ [11]. Energy gap is obtained by calculating the difference between the conduction band energy and the valence band energy, Eg = Ec-Ev so that we have Equation (2).

$$
E_{g}=\frac{\hbar^{2} \pi^{2} v_{f}^{2}}{9 w^{2} \gamma_{1}}
$$

The potential barrier for the p-n junction is shown in Figure 1 which is divided into 4 regions with boundary condition $\mathrm{z}=0, \mathrm{z}=\mathrm{d} / 2, \mathrm{z}=\mathrm{d}$. 


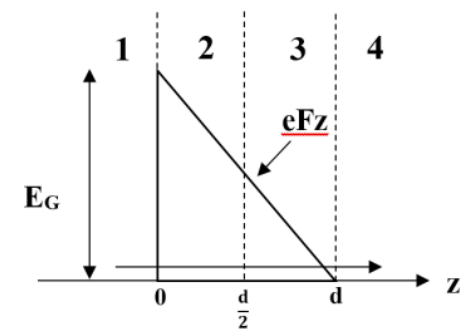

Fig. 1. Potential barrier in the depletion region of the p-n junction bilayer armchair graphene nanoribbon.

Mathematically, the potential profile above can be written like Equation (3).

$$
V(z)=\left\{\begin{array}{lr}
0, & z<0 \\
E_{g}-e F z, & 0 \leq z \leq d \\
0, & z>d
\end{array}\right.
$$

With Eg is energy gap, e is electron charge, $\mathrm{F}$ is electric field, and $\mathrm{z}$ is the position at depletion region. The wave equation at each region is in Equation (4).

$$
\begin{array}{rlrl}
\psi_{1} & =R e^{i k_{1} z}+S e^{-i k_{1} z}, & & z<d_{1} \\
\psi_{2} & =C A_{i}(\zeta(z))+D B_{i}(\zeta(z)), & & d_{1}<z<d_{2} \\
\psi_{3} & \left.=P A_{i}(\xi(z))+F B_{i} \xi(z)\right), & & d_{2}<z<d_{3} \\
\psi_{4}=G e^{i k_{2} z} & & z>d_{3}
\end{array}
$$

$A_{i}(\zeta(z))$ and $B_{i}(\zeta(z))$ is the Airy function of the first kind, while $A_{i}(\xi(z))$ and $B_{i}(\xi(z))$ is the Airy function of the second kind, with $\zeta(z)=\left(\frac{2 m_{1} e f}{\hbar^{2}}\right)^{1 / 3}\left(\frac{E_{g}-E}{e F}-z\right)$ and $\xi(z)=$ $\left(\frac{2 m_{2} e f}{\hbar^{2}}\right)^{1 / 3}\left(\frac{E_{g}-E}{e F}-z\right), \mathrm{E}$ is the electron energy, $m_{1}$ and $m_{2}$ is the effective mass electron in $\mathrm{p}$ type and n-type. Because the width of the depletion region is $\mathrm{d}$, so $d_{1}=0, d_{2}=\frac{d}{2}$, and $d_{3}=\mathrm{d}$. Then, constant R, S, C, D, P, F, and G are known by using boundary condition $\mathrm{z}=0, \frac{d}{2}$, dan $\mathrm{d}$. After all constants are known, the transmittance value is obtained through the Equation (5).

$$
T=\frac{k_{2}}{k_{1}}\left|\frac{G}{R}\right|
$$

The tunneling current can be calculated using the Equation (6) [13]

$$
I=\frac{2 g_{V} e}{h} \int_{0}^{e V_{b}}\left[f_{V}(E)-f_{C}(E)\right] T(E) d E
$$

$f_{V}(E)=\left(1+\exp \left[\left(E-e V_{b}\right) /\left(k_{B} T\right)\right]\right)^{-1}$ and $f_{c}(E)=\left(1+\exp \left[E /\left(k_{B} T\right)\right]\right)^{-1}$ are the Fermi-Dirac distribution function for the electron in the valence band and conduction band, $k_{B}$ is the Boltzman constant, $\mathrm{h}$ is the Planck constant, $g_{v}$ is degeneration of GNR $\left(g_{v}=1\right)$, and $T(E)$ is the transmittance as a function of energy. The calculation of tunneling current solved by gaussian quadrature. 


\section{Result and Discussion}

Transmittance as a function of electron energy is shown in Figure 2. The parameters used in this research are the index $\mathrm{N}=28(\mathrm{w}=3.32 \mathrm{~nm})$, the electric field $\mathrm{F}=1 \mathrm{MV} / \mathrm{cm}$, and the temperature $\mathrm{T}=300 \mathrm{~K}$ with electron energy $0.001-0.1 \mathrm{eV}$. The BAGNR energy gap calculation result is $0.11 \mathrm{eV}$ with a barrier potential width of $1.1 \mathrm{~nm}$.

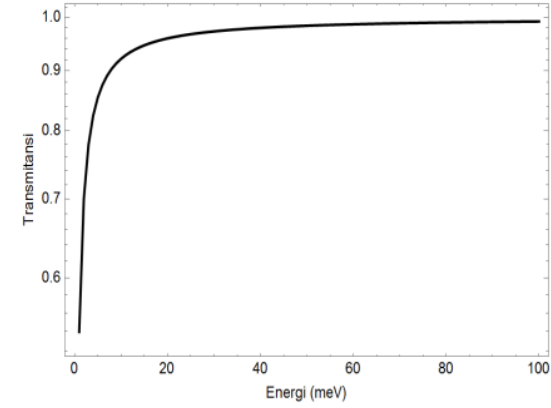

a)

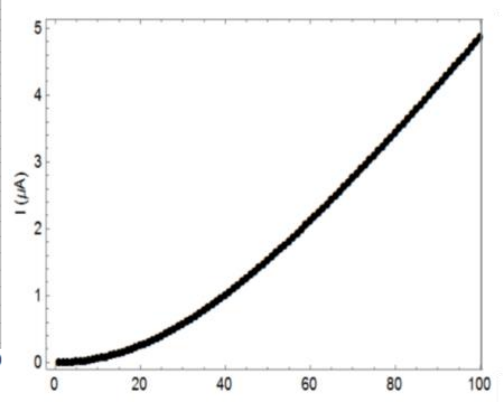

b)

Fig. 2. (a) Electron Transmittance on p-n junction bilayer armchair graphene nanoribbon, (b) Relation of the tunneling current to the bias voltage.

Based on the graph in Figure 2(a), electron transmittance has occurred at $0.001 \mathrm{eV}$ electron energy. This shows that even though the electron energy is lower than the barrier potential energy, electrons can still tunnel the barrier potential. In the case of $p-n$ junction, transmittance shows the probability of electrons successfully tunneling the barrier potential when moving from the p-type semiconductor valence band to the n-type conduction band [14],[16]. The increase of the electron energy causes the transmittance also increase. When the transmittance approaches its maximum value, the change in transmittance to the increase of electron energy will be smaller, this condition is called the saturation state. The triangle potential barrier shape causes the width of the potential barrier that must be penetrated by electrons in each energy value is not same. When electron energy be higher, the width of potential barrier that must be passed by electron will be smaller and make electron easier to penetrate the barrier potential. In addition, increasing electron energy also increases the ability of electrons to penetrate the potential barrier. In this research, $0.01 \mathrm{eV}$ electron energy can result the transmittance that is close to maximum so that the energy value above it does not provide such a large transmittance change.

Figure 2(b) shows a graph of tunneling currents as a function of a reverse bias whose value 1-100 $\mathrm{mV}$. In the equilibrium condition before reverse bias is applied, the p-type semiconductor valence band is at the same energy level as the n-type conduction band and no electron or holes flow through the junction region. The tunneling current increase with the increase in applied reverse bias. These results have the same tendency with previous research by [10] who model the tunneling current behavior in $\mathrm{p}$-n junction diodes that made from Monolayer Armchair Graphene Nanoribbon (MAGNR). When the reverse bias is applied, the high of the barrier potential will increase so that the p-type semiconductor valence band is above the n-type conduction band. As a result, the electron from the p-type valence band will tunnel to the n-type conduction band. The increase of bias voltage causes the p-type semiconductor valence band will be farther above the n-type conduction band so that the tunneling electron will be more copious. The application of the bias voltage will provide additional energy for electrons to tunnel 
the barrier potential. The number of electron that successfully penetrates the barrier potential is proportional to the value of the tunneling current. The diagram can be seen in Figure 3.

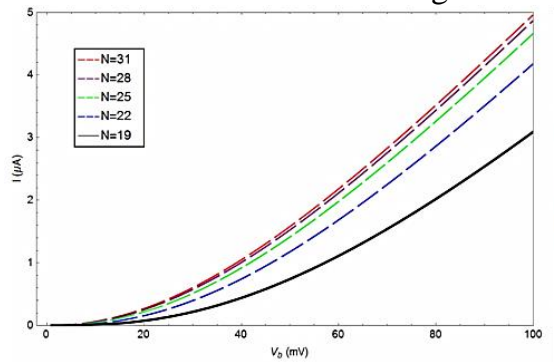

a)

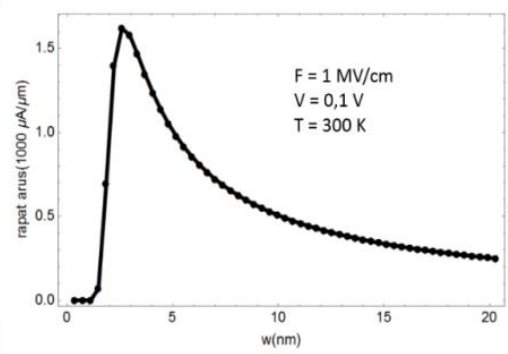

b)

Fig. 3. (a) Tunneling current with variation index N, (b) Density current to the width of BAGNR.

Figure 3(a) shows the effect of the index $\mathrm{N}$ to the tunneling current. The tunneling current increases with the increase of the index $\mathrm{N}$. Index $\mathrm{N}$ is the number of the atom that composes BAGNR. The $\mathrm{N}$ value determines the width and also the electrical properties of the BAGNR. The index $N$ that satisfy BAGNR as a semiconductor is $3 p+1$ with $p$ integer [17]. The width of the BAGNR is proportional to the $\mathrm{N}$ value. Based on research by [18],[17], the energy gap in GNR will be inversely proportional to its width, so that when $\mathrm{N}$ gets bigger, the energy gap is getting smaller. The energy gap will affect the depletion region width, which is proportional to the energy gap. When the energy gap gets smaller, the depletion region width becomes smaller. Tiny depletion region width will make it easier for an electron to penetrate it. From Figure 3(a) we can observe that for each increase of the index $\mathrm{N}$, the increase in the tunneling current will be smaller, as the increase in tunneling current that occurs from $\mathrm{N}=19$ to $\mathrm{N}=22$ is greater than the increase that occurred from $\mathrm{N}=22$ to $\mathrm{N}=25$, and so on. This happens because for each specified electric field and temperature value, the maximum current will be achieved at a BAGNR width value, as stated in [9]. The research's result by [9] regarding the effect of the graphene nanoribbon width to the tunneling current, for an electric field of $1 \mathrm{MV} / \mathrm{cm}$, the maximum current will be generated at a GNR width around $6.5 \mathrm{~nm}$ with a current density 450 $\mu \mathrm{A} / \mu \mathrm{m}$. Meanwhile, through the graph of current density to the width of BAGNR shown in Figure 3(b) we can see that the maximum current will be achieved at a width of BAGNR 2.58 $\mathrm{nm}(\mathrm{N}=22)$ with a current densityihure $1619.01 \mu \mathrm{A} / \mu \mathrm{m}$. We can see the graph in Figure 4.

The effect of temperature on the tunneling current is shown in Figure 4(a). Tunneling current decreases with the increase of temperature. The result of this research has the same tendency as the research's result by [9],[5]. The effect of temperature on tunneling current behavior can be explained through the Fermi-Dirac distribution function for electron in the valence band and the conduction band [10]. Temperature affects the resistivity and conductivity of a material. BAGNR, although it can be categorized as a semiconductor, the energy gap is very small and high charge mobility, causing its resistivity increase with increasing temperature, like a conductor. This is related to the vibrations that occur in the electron when the temperature is raised so that the electron collisions with other electron and it is difficult to arrive at a certain point. 


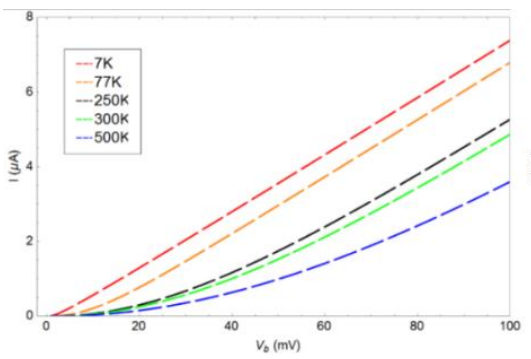

a)

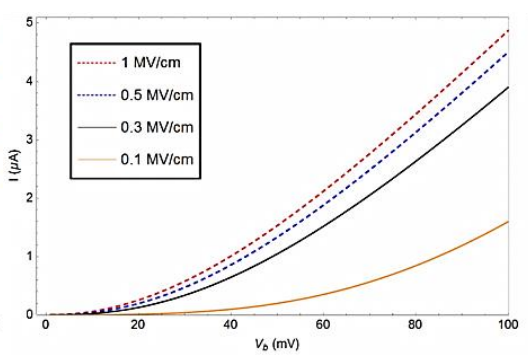

b)

Fig. 4. (a) Tunneling current with variation temperature, (b) Tunneling current with variation electric field.

Figure 4(b) shows the effect of the electric field to the tunneling current. The tunneling current increases with increasing electric field. The result of this research has the same tendency as the result of research by [13],[11],[10]. When the electric field gets bigger, the depletion region will be thinner so that electron is easier to tunnel the depletion region. The electric field in the depletion region can be designed based on the number of impurity atoms is given when making p-type and n-type semiconductor. The number of impurity atom will affect the charge polarization in the junction region that form the electric field [17]. The bigger electric field you want to produce, the dopping process has to be stronger, which means more impurities will be given. The more impurity atoms are given, the more carrier charge on the device and the tunneling current will increase. This can be seen in Figure 7.

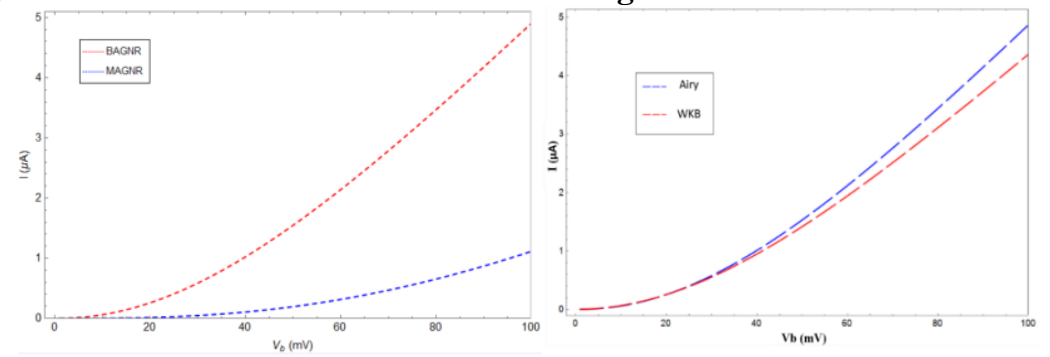

Fig. 7 a) shows the comparison between the tunneling current in the p-n junction bilayer armchair graphene nanoribbon (BAGNR) with monolayer armchair graphene nanoribbon (MAGNR). (b) shows a comparison of the tunneling current result using the Airy function approach method with the WKB method.

The tunneling current in the BAGNR material is bigger than the tunneling current in the MAGNR material. That is because the energy gap in the BAGNR is smaller than the energy gap in MAGNR. A smaller energy gap will have a smaller depletion region width so that the electron will be easier to tunnel. The calculation of the energy gap in MAGNR uses the equation is written in [12]. The structure of the energy band in BAGNR and MAGNR is affected by a hopping energy parameter between carbon atoms in graphene called interlayer coupling. The interlayer coupling can be determined by using the tight-binding model. The energy band on MAGNR is only influenced by $\gamma_{0}$, that connects the near neighbour carbon atoms in a graphene layer. Meanwhile, the energy band on BAGNR is influenced by $\gamma_{0}$ and $\gamma_{1}$, hopping energy parameter between atoms from one layer to another which position are parallel [20],[9],[6]. $\gamma_{1}$ has a direct effect on the effective mass value of the charge carrier on the bilayer graphene. 
Values $\gamma_{0}$ and $\gamma_{1}$ are used in the graph are $2.6 \mathrm{eV}$ and $0.3 \mathrm{eV}$, so that the energy gap BAGNR is $0.102 \mathrm{eV}$, while for MAGNR $0.349 \mathrm{eV}$. Comparison of the value of the effective mass and gap of energy bands between MAGNR and BAGNR as the effect of the parameters $\gamma_{0}$ and $\gamma_{1}$ are shown in the following Table 1.

Table 1. Comparison of the value of the effective mass and gap of energy bands between MAGNR and BAGNR.

\begin{tabular}{ccccccc}
\hline \multirow{2}{*}{$\boldsymbol{\gamma}_{\mathbf{0}}(\mathrm{eV})$} & \multirow{2}{*}{$\boldsymbol{\gamma}_{\mathbf{1}}(\mathrm{eV})$} & $\begin{array}{c}\boldsymbol{v}_{\boldsymbol{F}} \\
\end{array}$ & $\left(\times \mathbf{1 0}^{\mathbf{6}} \mathrm{m} / \mathrm{s}\right)$ & \multicolumn{2}{c}{$\boldsymbol{m}^{\mathbf{*}}$} & \multicolumn{2}{c}{$\boldsymbol{E} \boldsymbol{*}(\boldsymbol{e V})$} \\
\cline { 4 - 7 } & & & BAGNR & MAGNR & BAGNR & MAGNR \\
\hline \multirow{2}{*}{$3,12^{\mathrm{a}}$} & $0,3^{\mathrm{b}}$ & 1,01 & 2,36 & - & 0,146 & - \\
& $0,377^{\mathrm{a}}$ & 1,01 & 2,96 & - & 0,116 & - \\
$2,6^{\mathrm{b}}$ & $0,4^{\mathrm{c}}$ & 1,01 & 3,14 & - & 0,110 & - \\
$3^{\mathrm{c}}$ & & 0,84 & 3,39 & 3,95 & 0,102 & 0,349 \\
$3,12^{\mathrm{a}}$ & $0,3^{\mathrm{b}}$ & 0,97 & 2,55 & 3,43 & 0,135 & 0,403 \\
\hline & & 1,01 & 2,36 & 3,29 & 0,146 & 0,419 \\
\hline
\end{tabular}

$\mathrm{a}$ [21], b[22], c[23]

The result showed that the tunneling current from the Airy Function method and the WKB method has the same value at the beginning of the given bias voltage. When the voltage is increased and reaches a certain value, the tunneling current using the WKB method result a lower value than the Airy function. The result of this research has the same tendency with [12] that comparing the result of tunneling current in p-n junction made from Graphene Nanoribbon (GNR) using the Airy function approach and WKB method, see Equation (5). In calculations performed by [13], the solution of the wave equation using the WKB method becomes invalid at around and at $\mathrm{k}(\mathrm{z})^{2}=0$ (turning point).

$$
\frac{\mathrm{d}^{2}}{\mathrm{dx}^{2}} \psi(\mathrm{z})+\left(\frac{2 \mathrm{~m}}{\hbar^{2}}(\mathrm{E}-\mathrm{V}(\mathrm{z}))\right) \psi(\mathrm{z})=0
$$

with $\mathrm{k}(\mathrm{z})=\left(\frac{2 \mathrm{~m}}{\hbar^{2}}(\mathrm{E}-\mathrm{V}(\mathrm{z}))^{\frac{1}{2}}\right.$. Meanwhile, the Airy function approach can be valid in each region including the turning point. Based on this explanation, the difference in the tunneling current that occurs can be caused by electron energy approaching the turning point area so that at that voltage value, the tunneling current generated by the WKB method becomes less valid.

\section{Conclusion}

Based on the results of the study, the tunneling current increases with the increase in bias voltage, $\mathrm{N}$ index, and the electric field. Tunneling current decreases with increasing temperature. Tunneling current behavior in bilayer armchair graphene nanoribbon $p-n$ junction has the same tendency with monolayer armchair graphene nanoribbon and result in bigger tunneling current than monolayer armchair graphene nanoribbon. The Airy function method gives better results in calculating tunneling current than the WKB method because it gives valid values in each region including the turning point area. 


\section{Reference}

[1] Lee, J. U., Gipp, P. P., Heller, C. M.: Carbon nanotube p-n junction diodes. Appl. Phys. Lett. vol. 85. no. 1. pp. 145-147 (2004)

[2] Kim, S., Shin, D.H., Kim, C.O., Kang, S.S., Kim, J.M., Jang, C.W., Joo, S.S., Lee, J.S., Kim, J.H., Choi, S.H. and Hwang, E.: Graphene P-N Vertical Tunneling Diodes. ACS Nano. vol. 7. no. 6. pp. 5168-5174 (2013)

[3] Lau, C. N., Bao, W., Jr, J. V.: Properties of suspended graphene membranes. Mater. Today. vol. 15. no. 6. pp. 238-245 (2012)

[4] Suhendi, E.: Graphene dan Aplikasinya pada Divais Elektronika. Pros. Semin. Nas. Sains dan Teknol. Nukl., pp. 199-204 (2011)

[5] Suhendi, E., Syariati, R., Noor, F. A., Kurniasih, N.: Model of a tunneling current in a p-n junction based on armchair graphene nanoribbons - an Airy function approach and a transfer matrix method. vol. 91, pp. 1-5 (2014)

[6] Mccann, E., Koshino, M.: The electronic properties of bilayer graphene. vol. 056503 (2013)

[7] Zhang, Y., Tang, T.T., Girit, C., Hao, Z., Martin, M.C., Zettl, A., Crommie, M.F., Shen, Y.R. and Wang, F.: Direct observation of a widely tunable bandgap in bilayer graphene. Nature, vol. 459. no. 7248. pp. 820-823 (2009)

[8] Russo, S., Craciun, M.F., Koshino, M., Yamamoto, M., Tarucha, S. and Khodkov, T.: Electronic Transport Properties of Few-Layer Graphene Materials. INTECH Open Access Publisher (2011)

[9] Jena, D., Fang, T., Zhang, Q. and Xing, H.: Zener tunneling in semiconducting nanotube and graphene nanoribbon p-n junctions. Appl. Phys. Lett. vol. 93. no. 11. pp. 1-4, (2008)

[10] Yansen, W., Abdullah, M., Khairurrijal.: Application of Airy Function Approach to Model Electron Tunneling in Graphene Nanoribbon- Based P-N Junction Diodes. vol. 3. no. 1. pp. 3-6. (2010) [11] Syariati, R., Suhendi, E., Noor, F. A., and Khairurrijal. Simulation of Tunnel Current in an Armchair Graphene Nanoribbon-Based p-n Diode for Undergraduate Physics Students. Int. Conf. Adv. Educ. Technol. (ICAET 2014). no. Icaet. pp. 136-139 (2014)

[12] Fahmi, A.K., Hasanah, L., Rusdiana, D., Aminudin, A. and Suhendi, E.: Tunneling Current of Electron in Armchair Graphene Nanoribbon Bipolar Transistor Model Using Transfer Matrix Method. J. Phys. Conf. Ser., vol. 755. no. 1 (2016)

[13] Ghatak, A.K., Gallawa, R.L. and Goyal, I.C.: Modified Airy function and WKB solutions to the wave equation. NASA STI/Recon Tech. Rep. N (1991)

[14] Neto, A.C., Guinea, F., Peres, N.M., Novoselov, K.S. and Geim, A.K.: The electronic properties of graphene. Rev. Mod. Phys. vol. 81, no. 1. pp. 109-162 (2009)

[15] Suhendi, E., Syariati, R., Noor, F., Kurniasih, A. N.: Simulation of Dirac Tunneling Current of an Armchair Graphene Nanoribbon-Based p-n Junction Using a Transfer Matrix Method. vol. 974. pp. 205-209 (2014)

[16] Walker, J., Halliday, D., Resnick, R.: Fundamental of Physics. 10th ed (2014)

[17] Serway, R. A., Jewett, J. J W.: Physics For Scientists and Engineers with Modern Physics, 8th ed. USA (2010)

[18] Sze, S. M., Ng, K. K.: Physics of Semiconductor Devices. 3rd ed. John Wiley \& Sons, Inc. New Jersey (2007)

[19] Guo, J.: Modeling of Graphene Nanoribbon Devices. Nanoscale. vol. 4. no. 18. pp. 5538-5548 (2012)

[20] Kan, E., Li, Z., Yang, J.: Graphene Nanoribbons : Geometric, Electronic, and Magnetic Properties. Phys. Appl. Graphene-theory (2011)

[21] Chung, D. D. L.: Review Graphite. J. Mater. Sci. vol. 37. pp. 1475-1489 (2002)

[22] Min, H., Sahu, B., Banerjee, S. K., Macdonald, A. H.: Ab initio theory of gate induced gaps in graphene bilayers. Phys. Rev. B. pp. 1-7 (2007)

[23] Zhang, L. M., Li, Z. Q., Basov, D. N., Fogler, M. M., Hao, Z., Martin, M. C.: Determination of the electronic structure of bilayer graphene from infrared spectroscopy. Phys. Rev. B. pp. 1-11 (2008) 